\title{
Peroxisomal enzymes and 8-hydroxydeoxyguanosine in rat liver treated with perfluorooctanoic acid
}

\author{
Awad Abdellatif ${ }^{\mathrm{a}}$, Ahmad H. Al-tonsy ${ }^{\mathrm{b}, *}$, Mahmoud E. Awad ${ }^{\mathrm{c}}$, M. Roberfroid ${ }^{\mathrm{d}}$ and \\ M. Nezam Ullah Khan ${ }^{\mathrm{e}}$ \\ ${ }^{a}$ Garyounis Faculty of Medicine, Pharmacology Department, Benghazi, Lybia \\ ${ }^{\mathrm{b}}$ Benha Faculty of Medicine, Medical Biochemistry Department, Egypt \\ ${ }^{\mathrm{c}}$ Mansoura Faculty of Medicine, Forensic Medicine and Clinical Toxicology Department, Egypt \\ ${ }^{\mathrm{d}}$ Ecole de Pharmacie, Bruxelles, Belgium \\ ${ }^{\mathrm{e}}$ Garyounis Faculty of Medicine, Pathology Department, Benghazi, Lybia
}

\begin{abstract}
Although peroxisome proliferators are considered non-genotoxic agents, most of them, nevertheless, were found to promote and/or induce, hepatocellular carcinoma (HCC) in rodents. The aim of the present study is, first, to investigate whether the peroxisome proliferator perfluorooctanoic acid (PFOA) possesses inherent liver cancer promoting activity, and second, to study the possible mechanisms involved. To acheive these aims two protocols have been applied, a biphasic protocol (initiation by diethyl-nitrozamine (DEN) $200 \mathrm{mg} / \mathrm{kg}$ i.p. followed by treatment with $0.005 \%$ or $0.02 \%$ perflourooctanoic acid (PFOA) for 14 and 25 weeks) and a triphasic initiation, selection-promotion (IS) protocol (initiation by giving $200 \mathrm{mg} / \mathrm{kg}$ DEN i.p. followed by a selection procedure for 2 weeks consisting of giving $0.03 \%$-acetylaminofluorene (2-AAF) in diet). In the middle of this treatment a single oral dose of carbon tetrachloride $(2.0 \mathrm{ml} / \mathrm{kg})$ was given, followed by giving diet containg $0.015 \%$ of PFOA for 25 weeks. After applying both protocols, our results showed slight increase in the catalase activity while acyl CoA oxidase activity was markedly increased. Both experiments indicated that PFOA has a liver cancer promoting activity. Other groups of rats were given either basal diet or diet containing 0.02\% PFOA. Five or nine weeks later they were sacrificed and the levels of 8-hydroxydeoxyguanosine in the isolated DNA were estimated. The data showed a slight nonetheless insignificant increase in 8-hydroxydeoxyguanosine. From the present data, it is concluded that PFOA is a true liver cancer promoter that may not require extensive initial DNA damage for its promoting activity.
\end{abstract}

Keywords: Perfluorooctanoic acid (PFOA), diethylnitrosamine, (DEN), hepatocellular carcinoma (HCC), 2-acetylaminofluorene (2-AAF)

\section{Introduction}

Despite the fact that peroxisome hydrocarbons proliferators e.g. fibric acid derivatives, phthalates or perfluorinated hydrocarbons are neither mutagenic nor capable of provoking direct DNA damage, they are,

* Address for correspondence: Ahmad Hisham Al-tonsy, 10 Metwaly Noor Str., From Tayaran Str., Beside Dr. Abou El-azayem Hospital, Nasr City, Cairo, Egypt. Tel.: +2 024026748 or +2 0105251867; E-mail: Altonsi2000@yahoo.com. nonetheless, carcinogenic and also show promoting activity on rat liver cancer $[1,4,25,26,36]$. This lack of mutagenecity and/or direct DNA damaging activity led Reddy and associates to introduce the hypothesis that peroxisome proliferators may induce DNA damage by an indirect mechanism [26-28]. This notion is based on the realization that treatment of rodents with peroxisome proliferators is generally associated with a remarkable increase in peroxisomal $\beta$-oxidation of fatty acids as reflected by up to 30 -fold increase in fatty acyl CoA oxidase activity whereas that of catalase was 
marginally (up to 2.5 -fold) increased $[1,31,36]$. Consequently an imbalance between hydrogen peroxide $\left(\mathrm{H}_{2} \mathrm{O}_{2}\right)$ producing enzymes (e.g. acyl CoA oxidase) and $\mathrm{H}_{2} \mathrm{O}_{2}$ metabolizing enzyme (catalase) leads to substantial increase in $\mathrm{H}_{2} \mathrm{O}_{2}$ intracellular steady state concentration. This in turn could lead to the generation of chemically reactive oxygen species e.g. superoxide anion, singlet oxygen as well as hydroxyl radical, thereby initiating radical-mediate deleterious effects. If persists, this oxidative stress coupled with enhanced lipid peroxidation may participarte in extensive DNA damage and creates conditions favourable for neoplasmic development [14].

Kasai and co-workers [14] provided direct evidence for a relationship between peroxisome proliferation, oxidative stress and DNA damage. They observed significant increase in 8-hydroxydeoxyguanosine (8OHDG, a marker for hydroxyl radical-mediated DNA damage) in livers of rats kept on diet containing the peroxisome proliferator ciprofibrate for a protracted period. Perfluorooctanoic acid (PFOA) has been found to stimulate peroxisome proliferation $[1,3]$, induce apoptosis in human hepatoma HCPG2 cell through generation of reactive oxygen species [19] and promotes liver cancer development in rat liver [1]. The present investigation was designed to (a) further investigate the liver cancer promoting activity of perfluorooctanoic acid (PFOA) by different protocols. and (b) if so, what is the mechanism likely to be involved.

\section{Materials and methods}

\subsection{Animals}

Male Wistar rats, initially weighing between 180 and $200 \mathrm{~g}$, were used throughout. The animals were housed under standard conditions and were allowed free access to food and tap water. Minimum of 15 rats per group were tested.

\subsection{Chemicals}

Diethylnitrosamine (DEN) was obtained from Merk (Dramstad, Germany), 2-acetylaminofluorene (2-AAF), perfluorooctanoic acid (PFOA) were supplied by Aldrich (Beerse, Belgium) and carbon tetrachloride $\left(\mathrm{CCl}_{4}\right)$ was commercially available. Both 2acetylaminofluorene (2-AAF) and PFOA were admixed with a basal diet (BD) (Laboratory Rodent Diet, a Constant Nutrition ${ }^{\mathrm{TM}}$ formulation). DEN was dissolved in normal saline and administered via the i.p. route in a single dose of $200 \mathrm{mg} / \mathrm{kg}$. $\mathrm{CCl}_{4}$ was given in a single oral dose $(2 \mathrm{ml} / \mathrm{kg}$ in maize oil 1:1) as previously described by Neubauer et al. [18].

\subsection{Experimental procedures}

\subsubsection{Biphasic protocol}

The biphasic procedure for tumor initiation adopted in the present study was that initially reported by Peraino and others [21] and later modified by Pitot et al. [22]. Rats were divided into control and DEN treated (initiated "I" ) groups. The control group received normal saline $(2.0 \mathrm{ml} / \mathrm{kg}$ i.p) whereas I rats were treated with a single i.p injection of DEN $(200 \mathrm{mg} / \mathrm{kg})$. Two weeks later (time necessary to allow recovery from acute toxic effects of DEN) the rats were subgrouped and admitted to either BD or diet containing PFOA $(0.005 \%$ and $0.02 \%)$. This approach is designated as initiation "I". Fourteen and twenty five weeks after initiation the rats were sacrificed and their livers were used for biochemical and histological analysis.

\subsubsection{Triphasic protocol}

This procedure is identical to that previously reported by Lans et al. [16], as in the biphasic protocol, rats were treated with DEN (200 mg/kg i.p) for two weeks. After that, as a selection procedure, they were maintained for further two weeks on a diet containing 2AAF $(0.03 \mathrm{w} / \mathrm{w})$. After one week from starting the 2AAF treatment and in order to stimulate proliferation of DEN initiated cells, all animals received a single necrotizing oral dose of $\mathrm{CCl}_{4}(2.0 \mathrm{ml} / \mathrm{kg})$. After finishing the initiation selection procedure, rats were divided into different groups and were supplied with either BD or diet containing $0.015 \%$ of PFOA. In the present study we designated this protocol as initiation-selection "IS" procedure. Twenty five weeks later animals were sacrificed and their livers were used for biochemical and histological examination.

\subsubsection{Effect of PFOA on the level of 8-hydroxydeoxyguanosine in rat liver}

Rats were divided into 2 groups each of 20 animals, receiving either basal diet or diet containing $0.02 \%$ PFOA. Five and nine weeks later the rats were sacrificed, the livers were removed, the DNA was isolated and the level of 8-HODG was measured by HPLC equiped with electrochemical detector as previously described by Kasai et al. [14]. 


\subsubsection{Biochemical analysis}

Liver samples were homogenized in ice-cold $0.2 \mathrm{M}$ sucrose buffered with $3 \mathrm{mM}$ imidazole. The $\mathrm{pH}$ of the medium was adjusted to 7.4. The homogenates were used for estimation of enzyme activity.

\subsection{Measurement of enzyme activity}

\subsubsection{Catalase}

Catalase activity in the homogenates was determined according to the method of Baudhuin and associates [2]. Briefly, a $0.05 \mathrm{ml}$ of diluted liver homogenate was vortexed with $50 \mathrm{ul}$ of $2 \%$ triton. The mixture was then incubated at zero ${ }^{\circ} \mathrm{C}$ for $4 \mathrm{~min}$. After that we added $2.5 \mathrm{ml}$ solution containing $0.02 \mathrm{M}$ imidazole, $0.25 \mathrm{M}$ sucrose plus 0.1 bovine serum albumin and $105 \mathrm{mM}$ $\mathrm{H}_{2} \mathrm{O}_{2}$. The reaction was stopped by the addition of $1.5 \mathrm{ml}$ of a saturated solution of titanium sulphate in $2 \mathrm{~N}$ sulphuric acid and the remaining $\mathrm{H}_{2} \mathrm{O}_{2}$ was determined spectrophotometrically at $450 \mathrm{~nm}$. One unit of catalase activity was defined as the amount of enzyme causing the destruction of $90 \%$ of substrate in $1 \mathrm{~min}$, in a volume of $50 \mathrm{ml}$ under the assay conditions.

\subsubsection{Acyl CoA oxidase}

The activity of the peroxisomal acyl CoA oxidase was determined as previously described $[7,35]$. The measurement is based on the fluorimetric detection of $\mathrm{H}_{2} \mathrm{O}_{2}$. Briefly, in a total volume of $0.5 \mathrm{ml}$, the assay mixture containing $0.1 \mathrm{ml}$ of diluted liver homogenate, $0.02 \mathrm{mM}$ of flavine adenine dinucleotide, $80 \mathrm{mM}$ glycylglycine buffer (pH 8.3), $10 \mathrm{mM}$ homovanilic acid and 4 units of horseradish peroxidase. The reaction was started by the addition of $0.1 \mathrm{mM}$ palmitoyl CoA and fluorescence was measured spectrophotometrically at excitation and emission wavelengths of 325 and $426 \mathrm{~nm}$, respectively.

\subsubsection{Protein determination}

The protein concentration was estimated by the method of Lowery et al. [17], using bovine serum albumin as a reference standard.

\subsubsection{Histological analysis}

Rats were sacrificed by decapitation and their livers were excised and examined macroscopically for the presence of nodules which usually appear as white spots of 1-8 $\mathrm{mm}$ in diameter. Liver samples of $3 \mathrm{~mm}$ thickness were then taken and fixed in $10 \%$ formaline. Liver sections of 3-4 $\mathrm{mm}$ were prepared by the help of a microtome and then were stained by hematoxyline-eosin for subsequent microscopical examination.
Table 1

Catalase and acyl Co A oxidase (mU/mg protein) in rat liver 14 weeks after treatment with and without initiation

\begin{tabular}{llc}
\hline Treatment & Catalase & Acyl CoA oxidase \\
\hline Without inititation & & \\
BASAL DIET & $252 \pm 8.0$ & $3.47 \pm 0.1$ \\
PFOA (0.005) & $263 \pm 31.0$ & $26.25 \pm 5.0^{\mathrm{a}}$ \\
PFOA (0.02) & $334 \pm 8.0^{\mathrm{a}}$ & $50.00 \pm 9.0^{\mathrm{a}}$ \\
With initiation & & \\
BASAL DIET & $262 \pm 21$ & $2.95 \pm 0.1$ \\
PFOA (0.005) & $308 \pm 31^{\mathrm{a}}$ & $29.85 \pm 2.0^{\mathrm{a}}$ \\
PFOA (0.02) & $448 \pm 21^{\mathrm{a}, \mathrm{b}}$ & $78.25 \pm 7.0^{\mathrm{a}, \mathrm{b}, \mathrm{c}}$ \\
\hline
\end{tabular}

${ }^{\text {a }}$ Significant difference from basal diet.

${ }^{\mathrm{b}}$ Significant difference from 0.005 PFOA.

${ }^{\mathrm{c}}$ Initiation has a significant effect.

Table 2

Catalase and acyl Co A oxidase (mU/mg protein) in rat liver 25 weeks after treatment with and without initiation

\begin{tabular}{llc}
\hline Treatment & Catalase & Acyl coa oxidase \\
\hline Without inititation & & \\
BASAL DIET & $294 \pm 4.0$ & $5.0 \pm 0.5$ \\
PFOA (0.005) & $433 \pm 42.0^{\mathrm{a}}$ & $32.0 \pm 4.0^{\mathrm{a}}$ \\
PFOA (0.02) & $417 \pm 43.0^{\mathrm{a}}$ & $55.0 \pm 6.0^{\mathrm{a}}$ \\
With initiation & & \\
BASAL DIET & $261 \pm 7.0$ & $5.4 \pm 0.4$ \\
PFOA (0.005) & $263 \pm 27.0$ & $29.85 \pm 6.7^{\mathrm{a}}$ \\
PFOA (0.02) & $435 \pm 49.0^{\mathrm{a}}$ & $75.6 \pm 7.0^{\mathrm{a}}$ \\
\hline
\end{tabular}

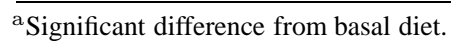

\section{Results}

\subsection{The effects of PFOA on liver catalase activity in initiated rats}

Data presented in Tables 1 and 2 show that induction in catalase activity by PFOA is significantly dependent on both dose and duration of treatment up to 1.5 fold. This was most evident in the group of rats which were sacrified 14 weeks after initiation with 2-AAF. Also in the same group initiation has found to increase the catalase inducing effect of PFOA, but it failed to show the same effect on the group sacrificed 25 weeks after initiation.

\subsection{The effects of PFOA on peroxisomal fatty acyl CoA oxidase activity in initiated rats}

To assess the extent of PFOA-induced $\mathrm{H}_{2} \mathrm{O}_{2}$ synthesis, we measured the increase in the activity of fatty acyl CoA oxidase. Data indicated in Tables 1 and 2 clearly show that induction of acyl CoA oxidase activity by PFOA was more pronounced than that of catalase. The effect was dose-related at 14 weeks. Data also indicate that I-pretreatment had significant $(P<0.05)$ effect on 
Table 3

Catalase and acyl Co A oxidase (mU/mg protein) in rat liver of the initiation-selection group

\begin{tabular}{lcc}
\hline Treatment & Catalase & Acyl CoA oxidase \\
\hline BASAL DIET & $239 \pm 16$ & $2.93 \pm 0.15$ \\
0.015 PFOA & $417 \pm 25^{\mathrm{a}}$ & $39.0 \pm 3.0^{\mathrm{a}}$ \\
\hline
\end{tabular}

${ }^{a}$ Significant difference from basal diet.

Table 4

Intrahepatic concentrations of 8-OH-deoxyguanosine in animals feed either basal diet (BD) or diet containing PFOA for 5 or 9 weeks

\begin{tabular}{lcc}
\hline Treatment & 8-OH-deoxyguanosine (ng/ug DNA) \\
\hline & 5 weeks & 9 weeks \\
BD & $0.126 \pm 0.04$ & $0.136 \pm 0.02$ \\
PFOA 0.02\% & $0.110 \pm 0.01$ & $0.149 \pm 0.05$ \\
\hline
\end{tabular}

the induction of the enzyme by the highest concentration used but only at 14 weeks post-initiation. Induction figures were 7.4 and 14-fold at 14 weeks and 10 and 11-fold at 25 weeks for rats treated with low and high dose of PFOA respectively.

\subsection{Effects of PFOA on liver catalase activity in IS- pretreated rats}

The results presented in Table 3 show that in animals subjected to IS-PFOA treatment, catalase activity was significantly $(P<0.05)$ increased by approximately 2.3-fold as compared to control (IS-BD) value.

\subsection{Effects of PFOA on liver fatty acyl CoA oxidase activity in IS- pretreated rats}

The data displayed in Table 3 indicate that IS-PFOA treatment resulted in marked induction of peroxisomal acyl CoA oxidase activity. The activity of the enzyme was increased by a factor of 24 in IS-PFOA treated rats when compared to control value.

\subsection{Effects of PFOA on 8-HODG liver concentration}

The effects of treatment with PFOA on liver concentration of 8-HODG (a marker for hydroxyl radicalmediated DNA damage) are illustrated in Table 4. Treatment of initiated rats with PFOA $(0.02 \%)$ for 5 and 9 weeks appears to be without any significant $(P<0.05)$ effect on hepatic levels of 8-HODG.

\subsection{Results of histological analysis (Table 5)}

Histological examination of liver samples from all groups shows no histological findings of hepatocellular carcinoma (HCC) in the control groups (groups on basal diet). On the other hand, histological findings of HCC (25 weeks after treatment) were evident in 14\%, $33 \%$ and $55 \%$ in the $\mathrm{I}-0.005 \%$, IS- $0.015 \%$ and I- $0.02 \%$ PFOA respectively.

\section{Discussion}

Hypolipidemic agents that had clearly induced peroxisomal proliferation and liver cancer in rodents, did not cause any clear cut enhancement in peroxisomal proliferation nor liver carcinoma in human [11]. Peroxisome proliferators, markedly induce the hydrogen peroxide producing enzyme (mainly acyl CoA oxidase) but slightly increase catalase an enzyme playing important role in hydrogen peroxide degradation [12,20, 29]. Chronic administration of peroxisome proliferators induces liver cancer in rodents [5,26,29]. This may be attributed to the sustained oxidative stress, produced by these chemicals in rodent hepatocytes due to an imblance in the production and destruction of hydrogen peroxide [24,29,30]. The potential role of PFOA in promoting liver cancer development noted in the current study (Table 1) is consistent with the hypothesis that peroxisome proliferators are carcinogenic by virtue of their notable capability to induce peroxisomal B-oxidation and consequent accumulation of hydrogen peroxide [29]. Although these experiments suggest that at least in rat, intracellular accumulation of hydrogen peroxide due to the chronic administration of PFOA may effectively play a role in promotion of liver cancer development, the evidence nevertheless is indirect and circumstantial. The main obstacle for obtaining direct evidence is the technical difficulty in measuring $\mathrm{H}_{2} \mathrm{O}_{2}$ turnover rate. The effect of peroxisome proliferators on the enzymes involved in the biogenesis and degradation of peroxisomal $\mathrm{H}_{2} \mathrm{O}_{2}$ have been mostly reported [12, 20,29]. Our results confirm these data and show that the induction of catalase by PFOA was dose dependent only when rats were sacrificed at the first, but not at the second sacrifice point and the induction of catalase by PFOA was more rapid in "I" pretreated than in control rats which magnify the possibility that "I" pretreatment modifies the induction pattern of PFOA. (see Figs 1 and 2). This confirms our previous reports on the hypolipidemic agent nafinopin [1]. Mechanism(s) 
Table 5

Effects of chronic treatment with PFOA on incidence of hepatocellular carcinoma in initiated and initiated selected rats

\begin{tabular}{|c|c|}
\hline Treatment & $\%$ Incidence of hepatocellular carcinoma \\
\hline $\mathrm{I}-\mathrm{BD}$ & Nil \\
\hline $\mathrm{I}-0.005 \%$ PFOA & $14 \%$ \\
\hline $\mathrm{I}-0.015 \%$ PFOA & $55 \%$ \\
\hline IS - BD & Nil \\
\hline IS- $0.015 \%$ PFOA & $33 \%$ \\
\hline IS $=$ Initiation selection & $\mathrm{BD}=$ Basal diet \\
\hline
\end{tabular}

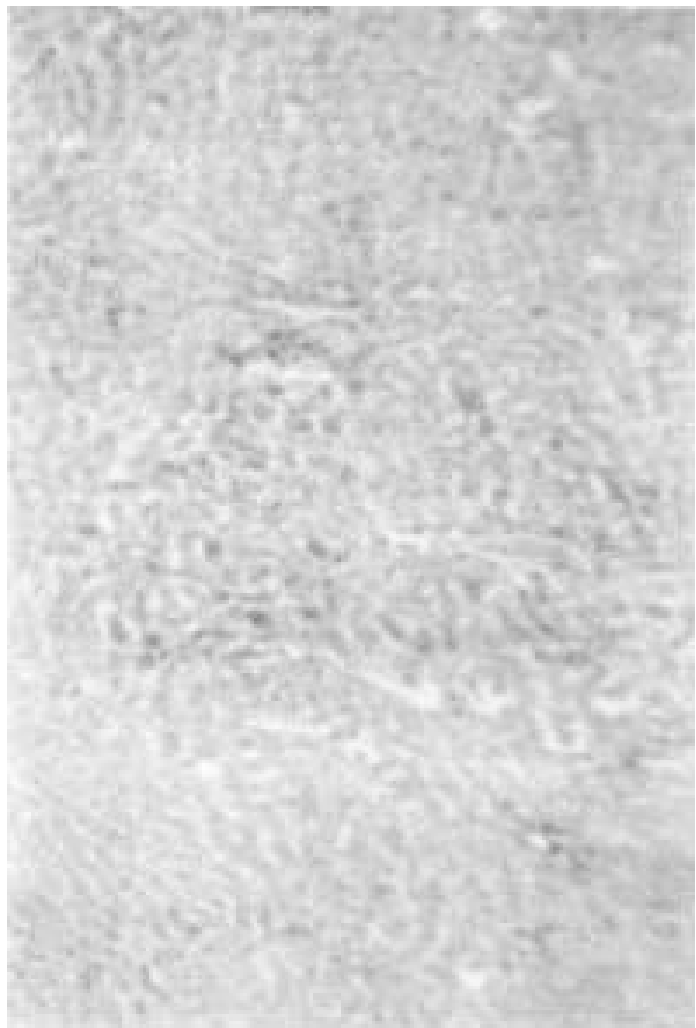

Fig. 1. Histological examination of a liver section from control animal shows normal liver.

by which PFOA induce catalase activity most probably by increase in enzyme synthesis rather than degradation. Reddy et al. [29] have shown that treatment of rats with a single dose of ciprofibrate (a peroxisome proliferator) provoked 1.4-fold increase in catalases mRNA estimated 1 and $16 \mathrm{~h}$. post-treatment.

The present results also reveal that feeding initiated animals with diet containing PFOA for 14 weeks markedly enhanced acyl CoA oxidase activity particularly at the highest concentration of PFOA. These data extends and compliments previous studies, that showed perfluoronated hydrocarbons have significant peroxisome proliferating activity (for review see [11]). Furthermore, the molecular mechanisms subserving

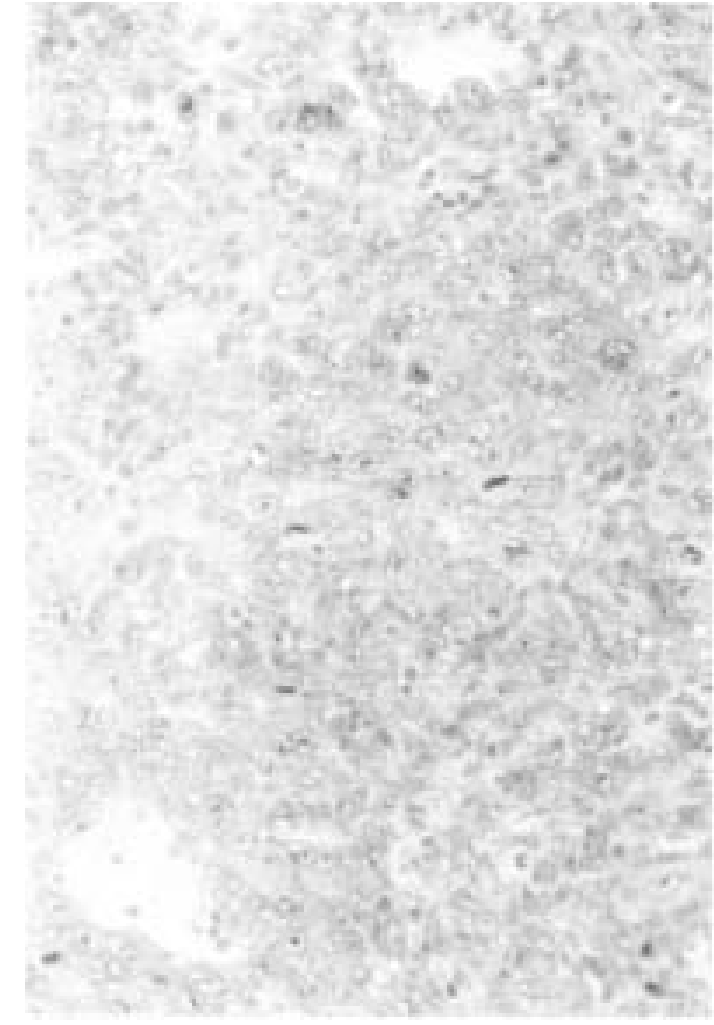

Fig. 2. Histological examination of a liver section from PFOA treated rat shows changes of hepatocellular carcinoma.

PFOA-mediated induction of fatty Acyl CoA oxidase may be related to the activation of peroxisome proliferator-activated receptor alpha (PPAR alpha) by PFOA [13].

Even though the carcinogenic effect of the peroxisome proliferators has been extensively studied, quite few investigations have dealt with the positive modulating aspects of these agents on liver carcinogenesis. Our data clearly show that PFOA produced positive modulating effect on rat liver carcinogenesis. In the same sense, the present data also reveal that PFOA appears to have more positive modulating activity than that previously reported for phenobarbitone $[9,23,33]$. Therefore, several trends are converging to lend sup- 
port to the notion that PFOA at the doses employed in the present study is a potent promoter and or modulator of liver cancer development. It might, therefore, be expected that PFOA promoting profile would involve an early DNA damage. Curiously, this predicted event did not materialize in both models for up to nine weeks treatment (Table 2). Despite the attraction of this hypothesis [8], there are several difficulties in recounciling it with some experimental data. First, earlier investigations have demonstrated that $\mathrm{H}_{2} \mathrm{O}_{2}$ generated by peroxisomes from animals pretreated with peroxisome proliferators was capable of producing double strand DNA breaks [8], second, Kasai et al. [14] have provided direct evidence for a close relationship between peroxisome proliferation, generation of reactive oxygen species and the hepatic levels of 8-HODG in rats kept on diet containing ciprofibrate for 18 months. Also, Takagi et al. [34] and Huang et al. [10] observed a siginficant increase in 8-OHDG level in cheer DNA of rats receiving perfluorinated fatty acid.

Consistent with the present finding, the observation of Elliott and Elcombe [6], that no significant liver DNA damage can be produced in rats kept for 4 weeks on a diet containing ciprofibrate or some other perixosomal proliferators, such as phthalate derivatives [15]. Also Kim et al. [15] found that the administration of perfluorodecanoic acid to rat for 8 weeks, did not produce any significant change in the level of 8-OHDG.

In conclusion, this study provide evidence that, similar to many other peroxisomal proliferators, PFOA possesses inherent, strong and specific inducing activity towards fatty acyl CoA oxidase and relatively weaker activity towards catalase. According to Reddy's hypothesis [29], this could create an imbalance between production and destruction of $\mathrm{H}_{2} \mathrm{O}_{2}$. Furthermore, the failure of PFOA to affect liver concentrations of 8OHDG in the present model (up to 9 weeks) do not exclude its indirect DNA damaging activity, but longer time may be needed in rats for peroxisome proliferators to produce significant DNA damage.

\section{References}

[1] A. Abdellatif, V. Preat, J. Vamecq, R. Nilsson and M. Roberfroid, Peroxisome proliferation and modulation of rat liver carcinogenesis by 2,4 dichlorophenoxyacetic acid, 2,4,5 dichlorophenoxyacetic acid, perfluoroocatanoic acid and nafinopin, Carcinogenesis 11 (1990), 1899-1902.

[2] P. Baudhum, H. Beaufay, Y. Rahman-Li, O. Sellinger, R. Wattiaux, P. Jacques and C. de Duve, Tissue fractionation studies: intracellular distribution of monoamine oxidase, aspartate aminotransferase, alanine aminotransferase, D-amino acid oxidase and catalase in rat liver tissue, Biochem. J. 92 (1964), 179-184.
[3] J. Berthiaume and K. Wallace, Perflourooctanoate, Perfluoroactanesulfate, and N-ethylperfluoroactanesulfanamidoethanol, peroxisome proliferation and mitochondrialbiogenesis, Toxicol. Lett. 129 (2002), 23-32.

[4] J. Christopher, P. Steven and S. Anya, General role of peroxisome proliferator-activated receptors in the actions of peroxisome proliferators, Ann. Rev. Pharmacol. Toxicol. 40 (2000), 491-518.

[5] J. Conway, R. Cattley, J. Popp and B. Butterwath, Possible mechanisms in hepatocarcinogenesis by peroxisome proliferator Di (2-ethylhexyl) phthalates, Drug Metabolism Rev. 21 (1989), 65-102.

[6] B. Elliott and C. Elcombe, Lack of DNA damage or lipid peroxidation measured in vivo in the rat liver following treatment with peroxisomal proliferators, Carcinogenesis $\mathbf{8}$ (1987), 1213-1218.

[7] G. Guilbaut, P. Brignac and M. Juneau, New substance for flurometric determination of oxidative enzyme, Anal. Chem. 40 (1968), 1256-1263.

[8] S. Goel, N. Lawani and J. Reddy, Peroxisome proliferation and lipid proxidation in rat liver, Cancer Res. 46 (1986), 13241330 .

[9] S. Horie and T. Suga, Different regulation of hepatic peroxisomal B-oxidation activity in rat treated with clofibrate and partialy hydrogenated marine oil, Biochem. Biophys. Res. Commun. 166 (1990), 780-786.

[10] C. Huang, M. Wilson, L. Lay, C. Chow, L. Roberson and H. Glanert, Increased 8-hydroxydeoxyguanosine in hepatic DNA of rats treated with the peroxisome proliferators ciprofibrate and perfluorodecanoic acid, Cancer Lett. 87 (1994), 223-228.

[11] W. Huber, B. Grasl-Kraupp and R. Schulte-Herman, Hepatocarcinogenic potential of di (2-ethylhexyl) phthalate in rodents and its implications on human risk, Crit. Rev. Toxicol. 26 (1990), 365-481.

[12] F. Ikeda, K. Aiba, K. Fukuda and M. Tanaka, The modulation of peroxisome poliferation in rat liver by perfluorinated fatty acids, metabolically inert derivatives of fatty acids, J. Biochem. 98 (1985), 475-482.

[13] U. Intrasuksri, M. Rangwala, M. O'Brien, J. Noonan and D. Feller, Mechanisms of peroxisome proliferation by perfluorooctanoic acid and endogenous fatty acids, Gen. Pharmacol. 31 (1998), 187-197.

[14] H. Kasai, Y. Okado, S. Nishimura, M. Rao and J. Reddy, Formation of 8-hydroxydeoxyguanosine in liver DNA of rats following long-term exposure to a peroxisome proliferator, Cancer Res. 49 (1989), 2603-2605.

[15] S. Kim, J. Hong, S. Janf, W. Kang, H. Yoo and Y. Yun, Formation of 8-oxodeoxyguanosine in liver DNA and hepatic injury by peroxisome proliferator clofibrate and perfluorodecanoic acid in rats, Toxicol. Sci. 23 (1989), 113-119.

[16] M. Lans, J. de-Gerlache, H. Taper, V. Prseat and N. Roberfroid, Phenobarbital as a promoter in the initiation-selection process of experimental rat hepatocarcinogenesis, Carcinogenesis 4 (1983), 141-144.

[17] O. Lowry, N. Rosebrough, A. Farr, R.J. Randall, Protein measurement with Folin phenol reagent, J. Biol. Chem. 193 (1951), 256.

[18] K. Neubauer, T. Knittel, T. Armbrust and G. Ramadori, Accumulation of fibrinogen/fibrin during short-term and long-term rat liver injury, Gastroenterology 108 (1995), 1124-1135.

[19] T. Panaretaki, I. Shabalina, D. Grander, M. Shoshan and J. De Pierre, Reactive oxygen species and mitochondria mediate the induction of apoptosis in human hepatoma HepG2 cells by rodent peroxisome proliferator and hepatocarcinogen, per- 
fluorooctanoic acid, Toxicol. Appl. Pharmacol. 173 (2001), 56-64.

[20] I. Pastoor, K. Lee, M. Perri and P. Gillies, Biochemical and morphological studies of ammonium perfluorooctanoateinduced hepatomegaly and peroxisome proliferation, Exp. Mol. Pathol. 47 (1987), 98-109.

[21] C. Peraino, R. Fry and E. Staffeldt, Reduction and enhancement by Phenobarbital of hepatocarcinogenesis induced in the rats by 2-acetylaminofluorene, Cancer Res. 31 (1971), 15061512 .

[22] H. Pitot, L. Bansness and T. Goldworthy, Biochemical characterization of stages of hepatocarcinogenesis after single dose of diethylnitrozamine, Nature 271 (1978), 456-458.

[23] V. Preat, J. de Gerlache, M. Lans, H. Taper and M. Roberfroid, Comparative analysis of the effect of phenobarbital, dichlorophenyltrichloroethane, butylated hydroxytoluene and nafenopin, Carcinogenesis 4 (1986), 1021-1029.

[24] M. Rao and J. Reddy, An overview of peroxisome proliferatorinduced hepatocarcinogenesis, Environ. Health Perspect. 93 (1991), 205-209.

[25] J. Reddy and S. Qureshi, Tumoriginicity of the hypolipidemic peroxisome proliferators, clofibrate in rat, Br. J. Cancer 40 (1979), 476-482.

[26] J. Reddy, D. Azarnoff and C. Hignite, Hypolipidemic hepatic peroxisome proliferators form a novel clew of chemical carcinogens, Nature 283 (1980), 397-398.

[27] J. Reddy, N.D. Lalwani, M. Reddy and S.A. Qureshi, Excessive accumulation of autofluorescent lipofusion in the liver during hepatocarcinogenesis by methylclofenapate and other hypolipidemic peroxisome proliferators, Cancer Res. 42 (1982), 259-266.

[28] J. Reddy and N.D. Lalwani, Carcinogenesis by hepatic peroxisome proliferators: evaluation of the risk of hypolipedemic drugs and industrial plasticizers to human CRC, Crit. Rev.
Toxicol. 12 (1983), 1-58.

[29] J. Reddy, S. Goel, M. Nemali, J. Carrino, T. Laffler, M. Reddy, S. Sperbeck, T. Osumi, T. Hashimoto, N. Lalwani and M. Roa, Transcriptional regulation of peroxisomal fatty acyl CoA oxidase and enoyl CoA hydratase and 3-hydroxyacyl CoA dehydrogenase in rat liver by peroxisome proliferators, Proc. Natl. Acad. Sci. 83 (1986), 1747-1751.

[30] J. Reddy and M. Rao, Oxidative DNA damage caused by persistent peroxisome proliferation: its role in hepatocarcinogenesis, Mutat. Res. 214 (1989), 63-68.

[31] M. Roberfroid, From normal cell to cancer: an overview introducing the concept of modulation of carcinogenesis, in: Concepts and Theories in Carcinogenesis, A. Meskens, P. Ebbesen and A. Burmy, eds, Elsevier Science, Amsterdam, 1987, pp. 157-167.

[32] K. Sai-kato, A. Takagi, T. Umemura, R. Hasegawa and Y. Kurokawa, Role of oxidative stress in non-genotoxic carcinogenesis with special reference to liver tumors induced by peroxisome proliferators, Biomed. Environ. Sci. 8(3) (1995), 269279.

[33] R. Schulte-Hermann, Tumor promotion in the liver, Arch. Toxicol. 57 (1995), 147-158.

[34] A. Takagi, K. Sai, T. Umemura, R. Hasegawa and Y. Kurokawa, Short-term exposure to the peroxisome proliferators, perfluorooctanoic acid and perfluorodecanoic acid, causes significant increase of 8-hydroxydeoxyguanosine in liver DNA of rats, Cancer Lett. 57 (1991), 55-60.

[35] J. Vamecq, F. Van-Hoof, Implication of a peroxisomal enzyme in the catabolism of glutamyl-CoA, Biochem. J. 221 (1984), 203-211.

[36] J. Ward, J. Rice, D. Creasia, P. Lynch and C. Riggs, Dissimilar patterns of promotion by di(2-ethylhexyl)phthalate and phenobarbital of hepatocellular mice carcinoma, Carcinogenesis 4 (1983), 1020-1029. 


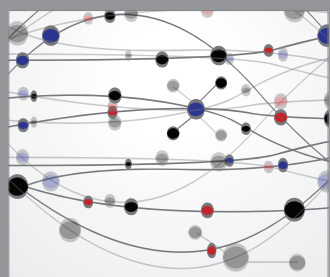

The Scientific World Journal
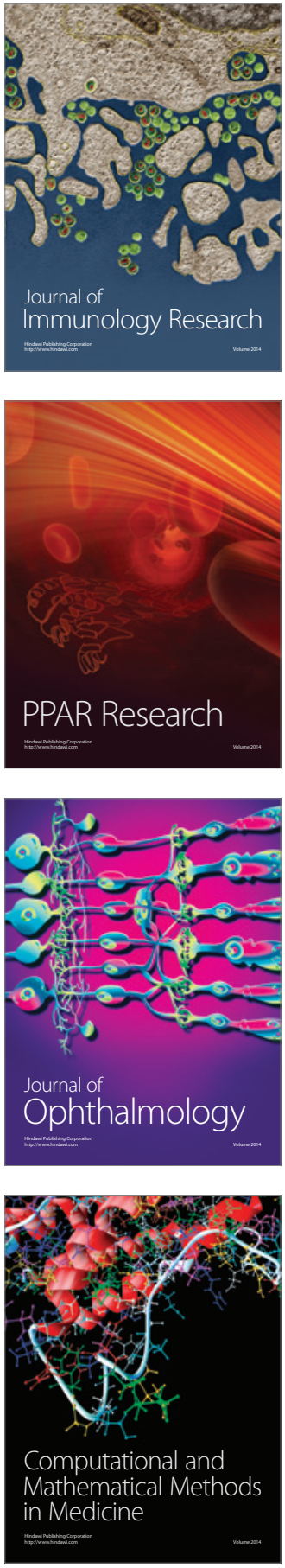

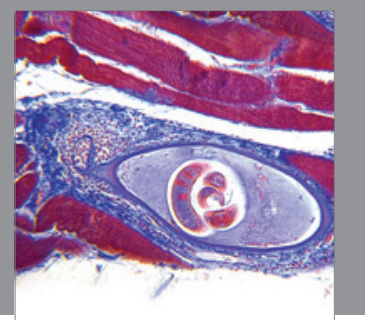

Gastroenterology

Research and Practice
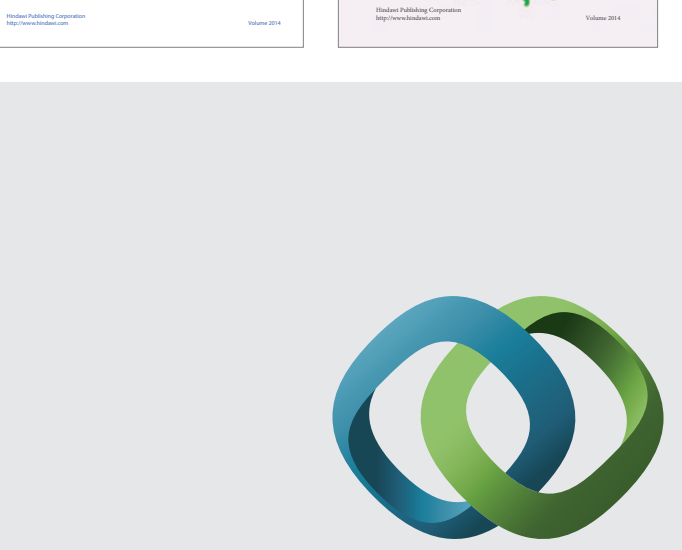

\section{Hindawi}

Submit your manuscripts at

http://www.hindawi.com
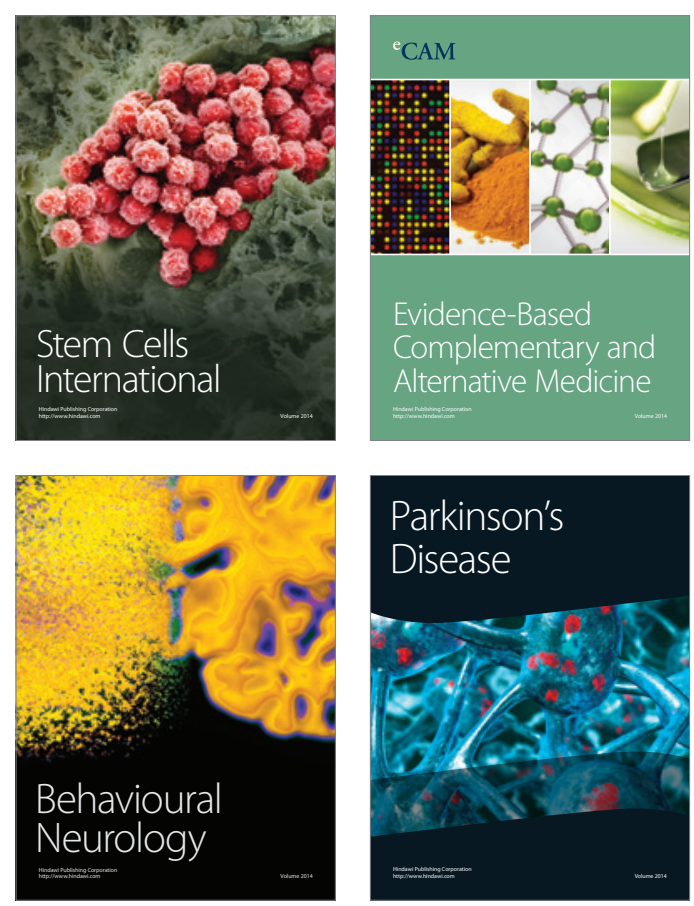

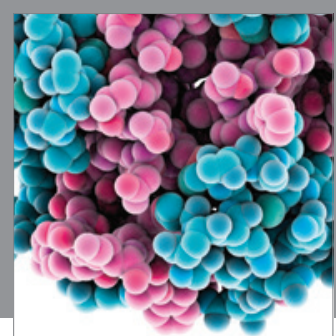

Journal of
Diabetes Research

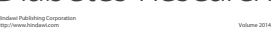

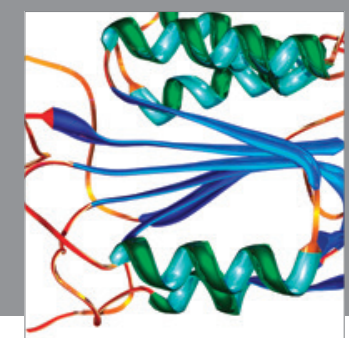

Disease Markers
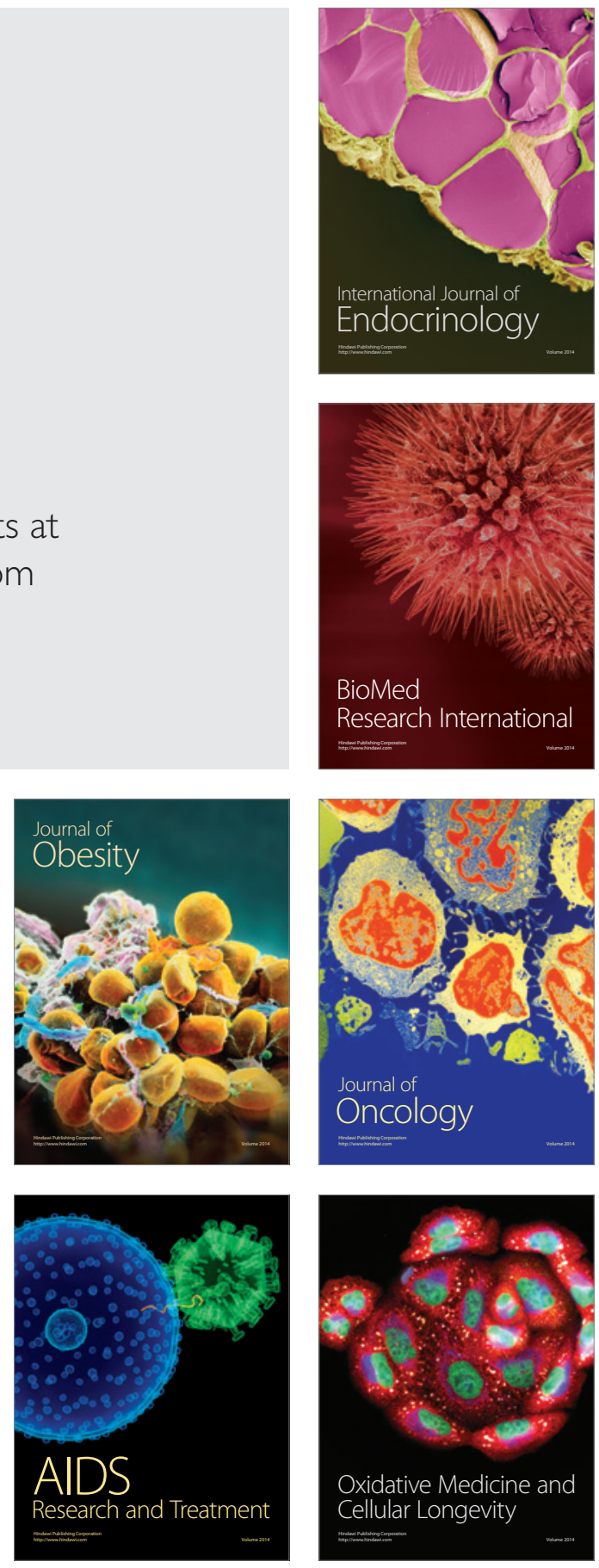